\title{
Correlación difusiva de la corriente de espín y electrones itinerantes en sistemas magnéticos confinados
}

\author{
Hernán Vivas-Calderón \\ Departamento de Física, Universidad Nacional de Colombia, Sede Manizales, Colombia
}

\begin{abstract}
Resumen
La interacción de las ondas de espín y los portadores electrónicos en un medio magnético es descrita desde la aproximación semiclásica de Landau-Lifshitz-Gilbert-Bazaliy (LLGB), adaptando el formalismo del propagador de difusión en sistemas infinitamente extendidos y confinados. La corrección estática y termodinámica de las fluctuaciones transversales en la susceptibilidad magnética es obtenida en términos de la densidad de corriente de portadores de carga $J_{e}$ y la intensidad del campo magnético externo $H_{0 Z}$, encontrándose una relación crítica del tipo $J_{e} \sim \mathrm{H}_{0 Z}^{1 / 2}$ en sistemas unidimensionales. La respuesta de la distribución de la densidad de portadores de espín en función de $J_{e}$, así como su correlación con la corriente de espín $\left(J_{S}\right)$ en estado estacionario, son discutidas para diferentes valores del campo aplicado y la diferencia de fase en la magnetización sobre las fronteras. El diagrama de fases asociado al tiempo de vida medio de la emisión de ondas de espín estimuladas térmicamente es calculado. El rôle del factor del Gilbert en la estabilidad térmica de los estados de ondas de espín es analizado cuantitativamente en este escenario.
\end{abstract}

Palabras claves: ondas de espín, densidad de corriente, propagador difusivo.

Diffusive Correlation for Spin Current and Itinerant Electrons in Confined Magnetic Systems

\begin{abstract}
Spin waves - electron carrier interaction in a magnetic medium is described from the Landau-Lifshitz-Gilbert-Bazaliy (LLGB) approximation, by adapting the diffusion propagator formalism on both infinitely extended and confined systems. The static and thermal correction due to the transverse fluctuations on the magnetic susceptibility are obtained in terms of the carrier density current $J_{e}$ and the externally applied field $H_{0 Z}$, providing a critical relationship giving by $J_{e} \sim \mathrm{H}_{0 Z}^{1 / 2}$, which is valid for $1 \mathrm{D}$ systems. The spin density distribution response as a function of $J_{e}$, as well as its correlation with the spin current $\left(J_{S}\right)$ in steady state, are discussed for different values of the applied field and boundary magnetization phase difference. Average lifetime phase diagram for thermally stimulated spin waves is also calculated. The Gilbert factor rôle on the thermal stability for the spin waves states is also quantitatively analyzed in this scenario.
\end{abstract}

Key words: Spin waves, current density, diffusive propagator.

\section{Introducción}

La espintrónica puede considerarse como un área interdisciplinaria muy activa que estudia las características singulares en la transferencia, transporte y acumulación del flujo de electrones con espín polarizado (Žutić I. et-al, 2004). El estudio de la fenomenología asociada a la interacción de espines electrónicos itinerantes en un medio magnéticamente ordenado ha ganado especial relevancia en la última década, aunque existen desarrollos teóricos importantes reportados desde los años 60 (Kondo J. 1964), (Hertz J., 1973) , (Berger L., 1996). La interacción mínima entre espines de diferentes especies es usualmente descrita a través del Hamiltoniano $s-d$, propuesto por Anderson (Anderson P., 1961) y definido por:

$$
\hat{\mathcal{H}}=-\mathcal{A s} \cdot \mathbf{S} \text {, }
$$

en donde $\mathbf{s}(\mathbf{S})$ corresponde al operador de espín de los electrones itinerantes (localizados) en el sistema, y $\mathcal{A}$ es una constante de acople. En el marco de la aproximación semiclásica, estos operadores toman los respectivos valores del momento de espín promedio (o densidad de espín electrónico) $\vec{\mu}=\langle\mathbf{s}\rangle$ y $\mathbf{S} \equiv S \mathbf{M} / M_{0}$, en donde $M_{0}$ es el máximo valor de la magnetización del sistema. En particular, el estudio de los fenómenos de contacto en la interfaz de una configuración de un metal no magnético $(\mathrm{N})$ y un ferromagneto (F), bajo la acción de una corriente inducida de espín desde $(\mathrm{F})$ hacia $(\mathrm{N})$ en el régimen de microondas, ha captado la atención de los investigadores en años recientes, dado que este tipo de arreglo permite inferir la relación entre corrientes de diferente naturaleza: la de portadores de carga y la de portadores del espín. Mencionaremos algunos casos específicos. La distribución espacial de la corriente de espín inyectada desde un sistema (YIG) hacia un metal (Pt) puede medirse utilizando técnicas propias del efecto Hall de espín inverso (ISHE) (Maekawa S., et-al, 2013). La correlación entre la densidad de corriente de carga $J_{C}$ y la corriente de espín inyectada en sistemas de tricapa del tipo $\mathrm{Pd} / \mathrm{Al} / \mathrm{Ni}_{80} \mathrm{Fe}_{20}$

\section{Correspondencia:}

Hernán Vivas-Calderón, hvivasc@unal.edu.co

Recibido: 12 de octubre de 2015

Aceptado: 15 de febrero de 2016 
también ha sido confirmada (Kitamura Y., et-al, 2013). En este caso, la precesión del campo de magnetización en la película ferromagnética genera una densidad de corriente de espín $J_{S}$ en el conductor metálico (Al). La densidad de corriente de portadores de carga medida en el Paladio (Pd) es producida por $J_{S}$ vía ISHE. Ondas de espín inducidas en películas de $\mathrm{CoFe} / \mathrm{Pd}-\mathrm{Cu}$ han sido caracterizadas utilizando la técnica de dispersión de Brillouin $\mu$-BLS (Madami M., et-al, 2011); y previamente, en un experimento de Resonancia del Espín Electrónico (ESR) en multicapas de $(\mathrm{Co} / \mathrm{Cu})_{N}$, considerado en ese entonces como una demostración realizable de la generación controlada de ondas de espín por emisión de radiación (SWASER) en el rango de 40 a $60 \mathrm{GHz}$ (Tsoi M., 2000). La observación directa del espectro de la radiación direccional del tipo BLS desde un arreglo de multicapas de $\mathrm{CuPyCuCo}{ }_{70} \mathrm{Fe}_{30} \mathrm{Au}$ en el intervalo de 7 a $11 \mathrm{GHz}$, constituye otra evidencia experimental del fenómeno de emisión de radiación de magnones producida por nano-osciladores (STNO) (Demidov V. E., 2010). Estos resultados incentivan la construcción de una base teórica conducente al cálculo de la respuesta de la corriente de espín en arreglos de tricapas del tipo Py/Metal/Metal (PMM), configuración en la cual se ha detectado recientemente el efecto Hall de espín inverso (Saitoh E. et-al, 2013). En este artículo se desarrollan las ecuaciones de Landau-Lifshitz-Gilbert-Bazaliy (LLGB) utilizando el formalismo del propagador de difusión y considerando la coexistencia de los fenómenos de transporte en $J_{e}$ y $J_{S}$ (Sección II). En la Sección (III) se discuten las propiedades de los modos de las ondas de espín en un sistema volumétrico, su longitud de coherencia, el tiempo de vida termodinámico y el propagador propio de la dinámica de las excitaciones colectivas modificadas bajo la acción de una fuente externa de corriente de portadores electrónicos de carga. En la Sección (IV) se calcula la relación de criticalidad entre la corriente y la magnitud del campo externo aplicado en sistemas unidimensionales. En el apéndice (I) se presenta una solución general utilizando el método de Dirichlet para condiciones de contorno arbitrarias en la densidad de espín.

\section{Formalismo LLGB: análisis en estado estacionario}

La ecuación generalizada de continuidad para la corriente de difusión del espín itinerante contiene una contribución del tipo torque $\mathbf{T} \propto \vec{\mu} \times \mathbf{M}$ sobre la evolución dinámica del campo M en un ferromagneto. Esta evolución es descrita desde la ecuación de Landau modificada, introduciendo los efectos de la interacción de la densidad de espín electrónico $\vec{\mu}$ sobre M (Zhang S., Li Z., 2010). Explícitamente:

$$
\frac{\partial \mathbf{M}}{\partial t}=-\gamma \mathbf{M} \times \mathbf{H}_{e f f}+\frac{\alpha}{M_{0}} \mathbf{M} \times \frac{\partial \mathbf{M}}{\partial t}+\mathbf{T},
$$

en donde $\mathbf{H}_{e f f}$ corresponde al campo magnético efectivo del tipo: $\mathbf{H}_{e f f} \approx \mathbf{H}_{0}+2 J \gamma^{-2}\left(\mathcal{K}+a_{m}^{2} \nabla^{2}\right) \mathbf{M} ; \mathcal{K}$ como el número de sitios más cercanos al punto $\mathbf{x}$ en donde se cal- cula la magnetización instantánea $\mathbf{M}(\mathbf{x}, t), a_{m}$ es el valor medio del parámetro de red del sistema magnético y $J$ define la interacción de intercambio entre primeros sitios (Phillips T. G., Rosemberg H. M., 1966). Estos términos son convenientemente normalizados a una constante de difusión del campo de magnetización $\mathcal{D}(J)=2 J M_{0} a_{m}^{5} / \hbar^{2} \gamma^{2}$. $\mathbf{H}_{0}$ denota el campo magnético externo aplicado. El término que contiene el factor $\alpha$ introduce el efecto de atenuación sobre $\mathbf{M}(\mathbf{x}, t)$ (Gilbert T. L., 2004). El torque inducido $\mathbf{T}$ en la geometría particular ilustrada en la Figura (1) se reduce a (Bazaliy Ya et-al, 1998), (Li Z., 2006), (Zhang S., 2009):

$$
\mathbf{T}=-\frac{b_{J_{e}}}{M_{0}^{2}} \mathbf{M} \times\left(\mathbf{M} \times \frac{\partial \mathbf{M}}{\partial z}\right)-\frac{c_{J_{e}}}{M_{0}} \mathbf{M} \times \frac{\partial \mathbf{M}}{\partial z},
$$

en donde los parámetros $b_{T_{e}}=\mu_{B} J_{e} / e M_{0} D_{0}$ y $c_{T_{e}}=$ $\left(\tau_{e x} / \tau_{S F}\right) b_{J_{e}}$ son directamente proporcionales a la magnitud de la densidad de corriente de conducción en la dirección $Z: \mathbf{J}_{e}=J_{e} \hat{\mathbf{z}}$. El factor $\tau_{S F}$ es tomado como el tiempo de relajación del espín localizado (spin flip), $\tau_{e x}$ es un parámetro característico del orden del inverso de la magnitud del acople entre los espines itinerantes y localizados $\hbar / \mathcal{A}$ [Ec. (1)], mientras que la densidad de corriente de espín $\mathbf{J}_{S}$ está relacionada con $\vec{\mu}$ a través de la ecuación de difusión de Fick: $\mathbf{J}_{\mu}^{v}=-D_{0} \nabla \mu^{v}$. La solución para $\vec{\mu}$ en la representación de las coordenadas circulares $\mu^{ \pm}=\mu^{X} \pm i \mu^{Y}$, puede obtenerse desde la expresión:

$$
D_{0} \nabla^{2} \vec{\mu}-\frac{1}{\tau_{e x} M_{0}} \vec{\mu} \times \mathbf{M}-\frac{1}{\tau_{S F}} \vec{\mu}=-\frac{\mu_{B}}{e M_{0}}\left(\mathbf{J}_{e} \cdot \nabla\right) \mathbf{M},
$$

y en el caso unidimensional:

$$
\frac{\partial^{2} \mu^{ \pm}}{\partial z^{2}}+\kappa_{1,2}^{2} \mu^{ \pm}=D_{0}^{-1} H_{J_{e}}^{ \pm}
$$

$\operatorname{con} m^{ \pm}=m^{X} \pm i m^{Y}$,

$$
H_{J_{e}}^{ \pm}=\frac{n_{0}}{M_{0}} \frac{\partial m^{ \pm}}{\partial t}-\frac{\mu_{B} J_{e}}{e M_{0} D_{0}} \frac{\partial m^{ \pm}}{\partial z}, \quad 0<z<\ell,
$$

y $\mathbf{M}(\mathbf{x}, t)=M_{0} \hat{\mathbf{z}}+\mathbf{m}(\mathbf{x}, t)$. El parámetro $n_{0}$ corresponde a la densidad de electrones, mientras que los valores de los coeficientes $\kappa_{i}$ están definidos por:

$$
\kappa_{1,2}=\left[ \pm i D_{0}^{-1}\left(\tau_{e x}^{-1} \pm i \tau_{S F}^{-1}\right)\right]^{1 / 2} .
$$

El término $\partial^{2} \mu^{ \pm} / \partial z^{2}$ es relevante en el caso en el cual se considera la dimensión de la pared de dominio suficientemente pequeña comparada con la longitud de difusión $\kappa^{-1}$, o cuando existen cambios abruptos de la magnetización en las interfaces en un sistema de multicapas. Los dos términos en la ecuación (5) pueden reconocerse directamente: el primero corresponde a las fluctuaciones temporales del campo $m^{ \pm}$y el segundo está asociado su variación espacial. Centraremos inicialmente nuestro 


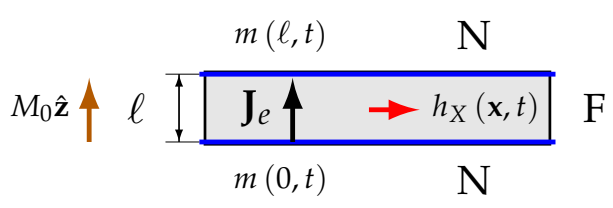

Figura 1. Sistema de una película ferromagnética (F) en contacto con electrodos metálicos no magnéticos $(\mathrm{N})$. La dinámica del campo de magnetización en la región $(\mathrm{F})$ inyecta corrientes de espín en (N) vía interacción del tipo $s-d$ en las interfaces.

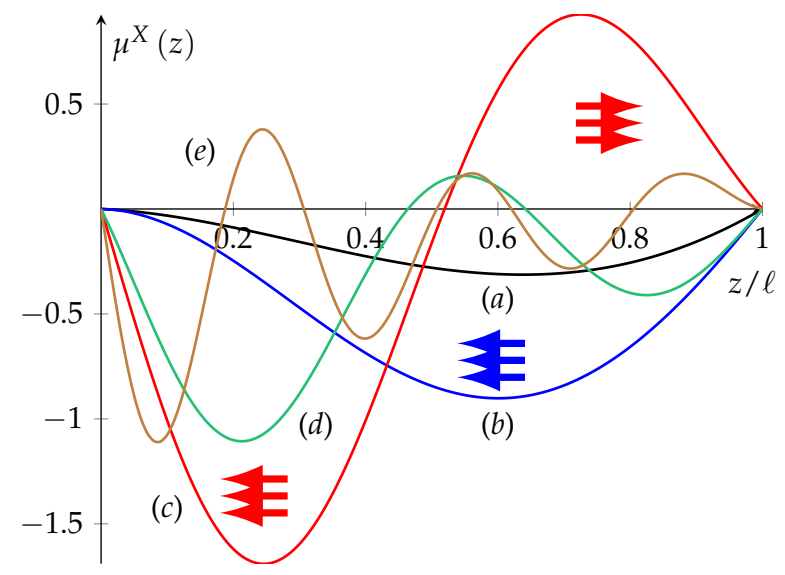

Figura 2. Distribución espacial de la densidad de espín electrónico $\mu^{X} \equiv \operatorname{Re}\left\{\mu^{+}\right\}$con $J_{e} / J_{e 0}$ dado por (a) 1 , (b) 2, (c) 3.5, (d) 5 y (e) 10. El campo magnético aplicado se define por $h_{0}=\tau_{S F} \gamma x^{-1} H_{0}=5$ y la diferencia de fase en los planos $z=0$ y $z=\ell$ de la película es $\Delta \phi=\pi$. La densidad de espín en los planos de contacto es cero. El parámetro $x=m_{0} / M_{0}$ define la relación de las amplitudes de la magnetización en el plano $X Y$ y perpendicular al mismo.

análisis en la condición $\partial m^{ \pm} / \partial t \rightarrow 0$, o en forma equivalente, bajo la consideración en la cual la dinámica de la magnetización es mucho más lenta que aquella asociada a la de los portadores electrónicos de transporte de carga. Las Figuras (2) y (3) ilustran las distribuciones de la densidad de espín para diferentes valores de la corriente de conducción $J_{e} / J_{S 0}$ y un valor fijo del campo magnético aplicado. El régimen de estado estacionario para el campo de magnetización puede interpretarse como la condición intrínseca de equilibrio que existe entre el torque magnético inducido por la corriente aplicada, $\mathbf{T}_{e}, \mathrm{y}$ el torque producido por la combinación del campo magnético externo y las fluctuaciones asociadas a la interacción de intercambio. En términos explícitos, $\mathbf{T}_{e}=\gamma \mathbf{M} \times \mathbf{H}_{e f f}$. La acumulación de la densidad de portadores de espín $\mu^{ \pm}$en las superficies se toman iguales a cero en primera instancia, aunque generalizaciones basadas en el criterio de irreversibilidad termodinámica han sido desarrolladas para interfaces en contacto del tipo F/F, F/N y N/N (Sears M. R., 2012). Existe un valor crítico de la corriente aplicada entre $2.0<J_{e} / J_{e 0}<3.5$ para el cual emerge un cambio en la orientación relativa de la densidad del es-

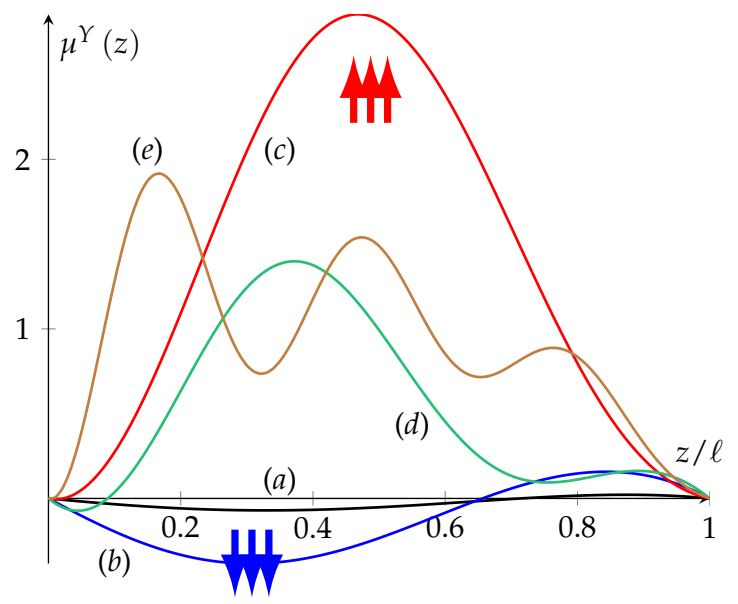

Figura 3. Distribución espacial de la densidad de espín electrónico $\mu^{Y} \equiv \operatorname{Im}\left\{\mu^{+}\right\}$con $J_{e} / J_{e 0}$ dado por (a) 1, (b) 2, (c) 3.5, (d) 5 y (e) $10 . h_{0}=\tau_{S F} \gamma x^{-1} H_{0}=5, \Delta \phi=\pi$.

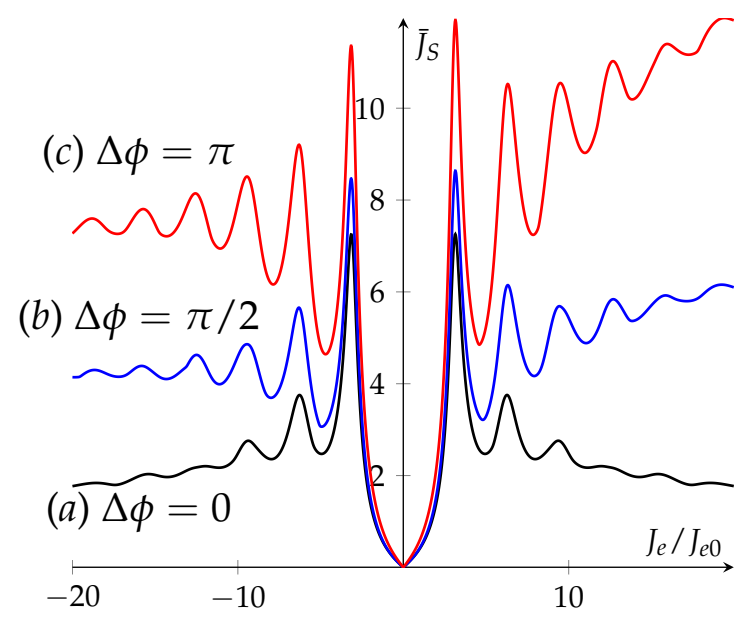

Figura 4. Valor medio de la corriente de espín $\bar{J}_{S}$ en función de la corriente de carga $J_{e}$ con $\mathcal{D}(J) \tau_{S F} / \ell^{2}=0.5$ y de fases relativas $\Delta \phi$. Las corrientes están normalizadas en los parámetros: $\bar{J}_{S}=$ $J_{S} / J_{S 0}, J_{S 0}=D_{0} n_{0} / \ell$. El campo exterior se define por $h_{0}=$ $\tau_{S F} \gamma x^{-1} H_{0}=5$.

pín electrónico [curvas (c)], mientras que un incremento en $J_{e}$ genera un patrón cuasiperiódico con longitud de onda $\lambda_{\mu}\left(J_{e}\right)$ el cual afecta el gradiente de la densidad del espín electrónico $\nabla \mu^{v}$, y a su vez, la corriente de espín $\mathbf{J}_{\mu}^{\nu}$. En el rango valores de $J_{e} \rightarrow 0$, y con campo externo aplicado cero, predomina la contribución de intercambio cuántico $2 J \gamma^{-2}\left(\mathcal{K}+a_{m}^{2} \nabla^{2}\right) \mathbf{M}$, mientras que los efectos de transporte electrónico se reflejan en las perturbaciones del perfil de la corriente de espín $J_{S}$. La correlación $J_{S}\left(J_{e}\right)$ es esencialmente de carácter lineal para valores de $J_{e} / J_{e 0}>10$, excepto en el caso simétrico en el cual la diferencia de fase en las fronteras de la película es cero: $\Delta \phi=0$ [Ver Figura (4)]. La densidad de corriente de referencia $J_{e 0}$ está definida por $J_{e 0}=e n_{0} D_{0}^{2} / \ell \mu_{B} x$. Las 


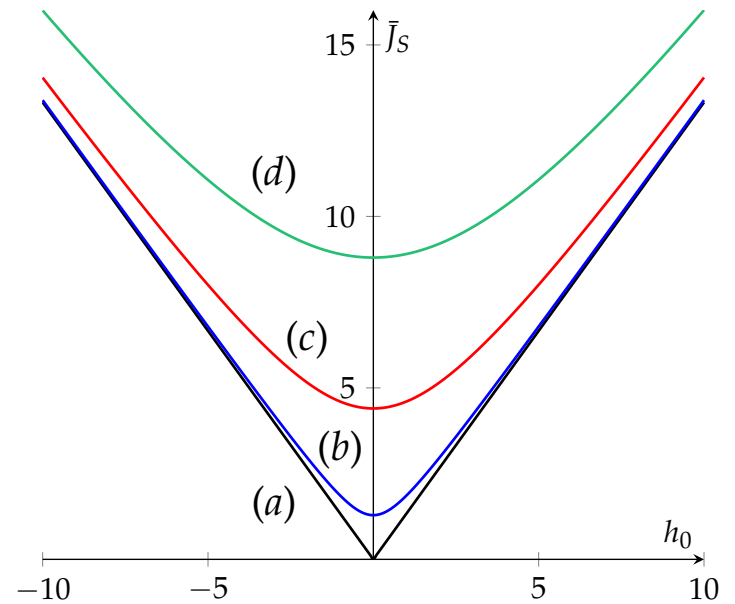

Figura 5. Corriente de espín en función del campo magnético aplicado con $J_{e} / J_{e 0}=3.3$ y $(a) \Delta \phi=0,(b) \Delta \phi=\pi / 4,(c)$ $\Delta \phi=\pi / 2,(d) \Delta \phi=\pi$.

variaciones de la oscilación en la corriente de espín en el régimen intermedio de valores de $J_{e} / J_{e 0}$ tienden a ser mayores para una diferencia de fase $\Delta \phi=\pi$, mientras que para valores pequeños de $J_{e} / J_{e 0}\left(J_{e} / J_{e 0}<2\right), \bar{J}_{S}$ exhibe un incremento monótono en su perfil hasta cierto valor crítico en donde la respuesta de la corriente de difusión presenta un carácter oscilatorio en su amplitud. La densidad de corriente de espín promedio en función del campo magnético transversal aplicado, con diferentes magnitudes de $J_{e} / J_{e 0}$, es representada en la Figura (5). $\bar{J}_{S}$ aumenta uniformemente con el incremento de $h_{0} \mathrm{y}$ $J_{e} / J_{e 0}$, observándose una fuerte dependencia del valor de la corriente de espín con la asimetría de la polarización de la magnetización en las superficies, y en ausencia del campo externo.

\section{Ondas de espín: longitud de propagación y tiempo de vida}

Una primera aproximación al cálculo de los modos de magnetización transversal puede obtenerse considerando que un campo magnético de prueba con polarización $\mathbf{h}(\mathbf{x}, t)=\left(h_{0 X} \exp \left[i\left(q_{X} x+q_{Z} z-\omega t\right)\right], 0, H_{0 Z}\right)$ $\mathrm{y}$ frecuencia $\omega$ incide sobre el sistema generando una magnetización $\left(m^{X}, m^{Y}, M_{0 Z}\right)$, definida a través de las componentes de la susceptibilidad $\chi_{i j}: m^{X}=\chi_{a} h^{X}$; $m^{Y}=-\chi_{b} h^{X}$. Los campos varían temporalmente según $\exp [-i \omega t]$, y la solución físicamente plausible corresponde a la condición $\operatorname{Im}\{\omega\}<0$. Las ecuaciones extendidas para el caso tridimensional se modifican desde la relación

$$
\hat{L}^{ \pm} m^{ \pm}=i \omega_{M} h^{ \pm}
$$

con el operador $\hat{L}^{ \pm}$definido por:

$$
\begin{aligned}
\hat{L}^{ \pm}=\mp & (1 \mp i \alpha) \frac{\partial}{\partial t}-i \mathcal{D}(J) \nabla^{2} \\
& -i\left(c_{J_{e}} \pm i b_{J_{e}}\right) \frac{\partial}{\partial z}+i \omega_{H},
\end{aligned}
$$

y $\omega_{M}=\gamma M_{0 Z}, \omega_{H}=\gamma H_{0 Z}$. Bajo la condición $\nabla$. $(\mathbf{h}+\mathbf{m})=0$, se obtiene $\left(1+\chi_{a}\right) q_{X}=0$, el cual tiene una solución del tipo $\chi_{a}=-1$ (Cottam M., 1989) ${ }^{1}$. El propagador asociado a la ecuación (8) toma la forma:

$$
K_{\infty}^{3 D \pm}\left(\mathbf{r}, \mathbf{r}^{\prime} ; t\right)=\frac{1}{8\left(\pi a_{ \pm}^{2} t\right)^{3 / 2}} \exp \left[-\frac{G^{ \pm 3 D}\left(\mathbf{r}, \mathbf{r}^{\prime} ; t\right)}{4 a_{ \pm}^{2} t}\right]
$$

con

$$
G^{3 D \pm}\left(\mathbf{r}, \mathbf{r}^{\prime} ; t\right)=\left(\left|\mathbf{r}-\mathbf{r}^{\prime}\right|+b_{ \pm} t\right)^{2}-4 a_{ \pm}^{2} t^{2} \bar{\omega}_{H^{\prime}}^{ \pm}
$$

$a_{ \pm}^{2}=\mp i \mathcal{D}(J) /(1 \mp i \alpha), b_{ \pm}=\left(b_{J_{e}} \mp i c_{J_{e}}\right) /(1 \mp i \alpha) \mathrm{y}$ $\bar{\omega}_{H}= \pm i \omega_{H}^{ \pm} /(1 \mp i \alpha)$. El cálculo de la expresión (9) es discutido en el Apéndice II. Las funciones $2 \chi_{a}=\chi_{q}^{+}+$ $\chi_{q}^{-} \mathrm{y}-2 i \chi_{b}=\chi_{q}^{+}-\chi_{q}^{-}$tienen la forma explícita en el dominio de la frecuencia y del momentum:

$$
\chi_{q}^{+}=\frac{i \omega_{M}}{\Delta_{q}^{+}}, \quad \chi_{q}^{-}=\frac{i \omega_{M}}{\Delta_{q}^{-}}
$$

$\operatorname{con} \Delta_{q}^{ \pm}= \pm i \omega(1 \mp i \alpha)+B_{q} \pm i b_{J_{e}} q_{Z}+i \omega_{H}$. La frecuencia de los modos de ondas de espín se obtiene desde la condición $\Delta_{q}^{ \pm}=0(\alpha=0)$ y poseen una dependencia con la corriente aplicada $J_{e}$ de la forma:

$$
\omega_{q}=-b_{J_{e}} q_{Z} \pm \sqrt{\bar{\omega}_{H q}\left(\bar{\omega}_{H q}+\omega_{M}\right)},
$$

$\operatorname{con} B_{q}=c_{J_{e}} q_{Z}+i \mathcal{D}(J)\left(q_{X}^{2}+q_{Z}^{2}\right)$ y $\bar{\omega}_{H q}=\omega_{H}-i B_{q}$. En el límite de las excitaciones en el contínuo $(q \rightarrow 0)$ y en ausencia de una corriente externa, la expresión (12) se reduce al resultado clásico de Damon-Eshbach (DE) (Eshbach J. R., 1960): $\omega_{D E}=\sqrt{\omega_{H}\left(\omega_{H}+\omega_{M}\right)}$. La longitud de propagación de las ondas de espín es definida por $\ell_{p}^{-1}=2 k^{\prime \prime}, \operatorname{con} k^{\prime \prime}=\operatorname{Im}\left\{\omega_{q} / v_{g}\right\}$, en donde $v_{g}$ es la velocidad de grupo de las excitaciones colectivas: $\partial \omega_{q} / \partial q_{Z}$. Los procesos de relajación de los portadores de espín en medios conductores limitan la longitud de coherencia de las ondas de tipo SFW (Spin Flip Waves), afectando a su vez las propiedades de transporte o control de la información lógica. En consecuencia, es de interés identificar

\footnotetext{
${ }^{1}$ Desarrollando el operador divergencia $\nabla \cdot(\mathbf{h}+\mathbf{m})=0$, $\left(1+\chi_{a}\right)\left(\partial_{X} h_{X}+\partial_{Y} h_{Y}\right)+\chi_{b}\left(\partial_{X} h_{Y}-\partial_{Y} h_{X}\right)=0$. En el caso particular con $q_{Y}=0$ y $h_{Y}=0$, la solución de la ecuación anterior se reduce a $\chi_{a}=-1$.
} 
los máximos de $\ell_{p}$ y sus posibles mecanismos de control vía campo externo $\left(\omega_{H}\right)$ o corriente aplicada $\left(J_{e}\right) \cdot \ell_{p}$ es calculado para modos no evanescentes ó $\operatorname{Im}\left\{\omega_{q}\right\}=0[\mathrm{Fi}-$ gura (6)]. La dependencia de la longitud de propagación de ondas de espín con el vector de onda $\ell_{p}\left(q_{z}\right)$ está en concordancia con el resultado experimental obtenido desde la técnica de dispersión Raman electrónica resonante (ERRS), aplicada sobre un gas electrónico bidimensional de pozos cuánticos del tipo $\mathrm{Cd}_{1-x} \mathrm{Mn}_{x} \mathrm{Te} / \mathrm{Cd}_{0.8} \mathrm{Mg}_{0.2} \mathrm{Te}$ (Gómez J. et-al, 2011). Los efectos de la intensidad de la corriente de portadores y el campo aplicado en la estructura de bandas para ondas de espín transversales se muestra en la Figura (7). La región de las frecuencias permitidas para $J_{e}=0$ se denota con $(A)$ y su ancho de banda tiende a disminuir para valores de $\bar{q}_{Z} \rightarrow \pi / 2$. Cuando la corriente es aplicada en dirección $+\mathbf{z}$ (banda $B)$, las frecuencias límites de los modos permitidos yacen en un intervalo de menor valor comparadas con aquellas asociadas a la banda $(A)$ con valores relativamente constantes en el régimen de grandes longitudes de onda. En el caso de corriente aplicada en dirección $-\hat{\mathbf{z}}$ (banda $C)$, emerge una región de frecuencias prohibidas de baja energía para $\bar{q}_{Z} \lesssim 0.5$, la cual puede interpretarse como la aniquilación de los estados de onda de espín producida por el incremento de la componente del torque de oposición $\mathbf{T}^{ \pm}$sobre la magnetización transversal $m^{ \pm}$(Eq. 3). Las componentes transversales de la susceptibilidad (11) pueden re-escribirse en el marco del formalismo de Dyson, en particular, desde el concepto de autoenergía propia: $\Sigma_{ \pm}^{\star}$.

$$
\chi_{q}^{ \pm}= \pm \omega_{M} \check{\mathbf{G}}_{q}^{ \pm}= \pm \omega_{M}\left(\omega \pm \hbar^{-1} \varepsilon_{\mathbf{q}}^{0}-\Sigma_{ \pm}^{\star}\right)^{-1}
$$

$\operatorname{con} \varepsilon_{\mathbf{q}}^{0}=\hbar \mathcal{D}(J) q^{2}$ y $\Sigma_{ \pm}^{\star}= \pm i \omega \alpha \pm i c_{J_{e}} q_{Z} \mp \omega_{H}-b_{J_{e}} q_{Z}$. Esta técnica permite calcular el tiempo característico de relajación de las excitaciones colectivas desde la prescripción:

$$
\tau_{\mathbf{q}}^{-1}=-\frac{\left.2 \operatorname{Im} \Sigma^{\star}\right|_{\varepsilon_{\mathbf{q}} / \hbar}}{1-\left.\frac{\partial}{\partial \omega} \operatorname{Re} \Sigma^{\star}\right|_{\varepsilon_{\mathbf{q}} / \hbar}}
$$

en donde las componentes real e imaginaria de $\Sigma^{\star}$ deben evaluarse para valores de frecuencias definidos por la energía renormalizada $\varepsilon_{\mathbf{q}}=\varepsilon_{\mathbf{q}}^{0}+\operatorname{Re}\left\{\hbar \Sigma^{\star}\right\}$ (Fetter A., 2002). El promedio del inverso del tiempo de vida relativo a la emisión de ondas de espín puede calcularse utilizando el resultado en el marco de la aproximación de Maxwell-Boltzmann (Chupis I. E., 1964), (Akhiezer I. A. et-al, 1961) :

$$
\left\langle\tau^{-1}\right\rangle=\frac{\Omega^{-1} \int \tau_{\mathbf{q}}^{-1} \exp \left(-\beta \varepsilon_{\mathbf{q}}\right) d^{3} \mathbf{q}}{\Omega^{-1} \int \exp \left(-\beta \varepsilon_{\mathbf{q}}\right) d^{3} \mathbf{q}},
$$

con $\beta^{-1}=k T$. El denominador del lado derecho de la ecuación (15), con $\varepsilon_{\mathbf{q}}=\varepsilon_{\mathbf{q}}^{0}+\hbar \omega_{H}-\hbar b_{J_{e}} q_{Z}$, corresponde al promedio termodinámico del número de estados excitados de ondas de espín $\langle n\rangle$ a temperatura $T$ y volúmen

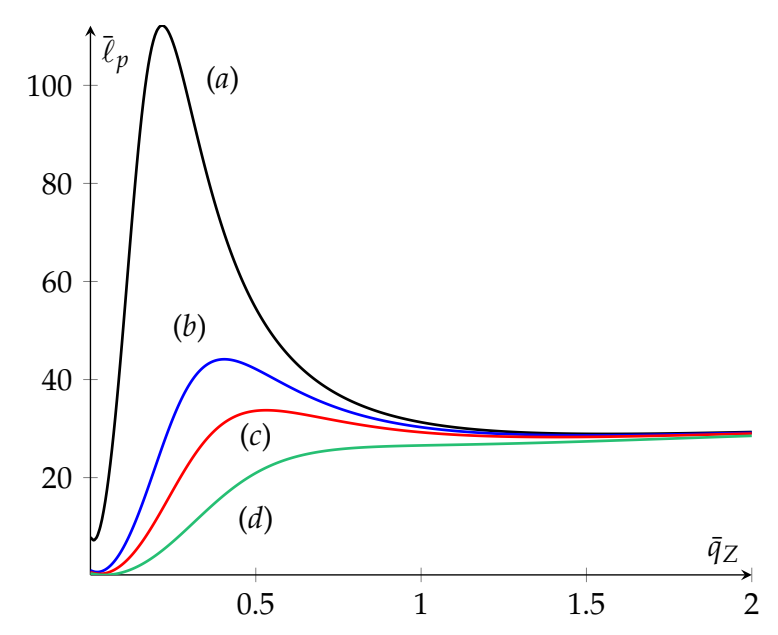

Figura 6. Longitud de propagación de las ondas de espín en función del vector de onda $q_{Z}$ para diferentes valores de $\omega_{h}$, con los valores normalizados $\bar{\ell}_{p}=\ell_{p} / a_{m}, \omega_{m} / \omega_{D E}=10$, $\mathcal{D}(J) / \omega_{D E}=5, b_{I_{e}} / \omega_{D E}=1, c_{I_{e}} / \omega_{D E}=0.3$. (a) $\omega_{h} / \omega_{D E}=$ 0.01 , (b) $\omega_{h} / \omega_{D E}=0.1$, (c) $\omega_{h} / \omega_{D E}=0.2$, (d) $\omega_{h} / \omega_{D E}=0.5$.

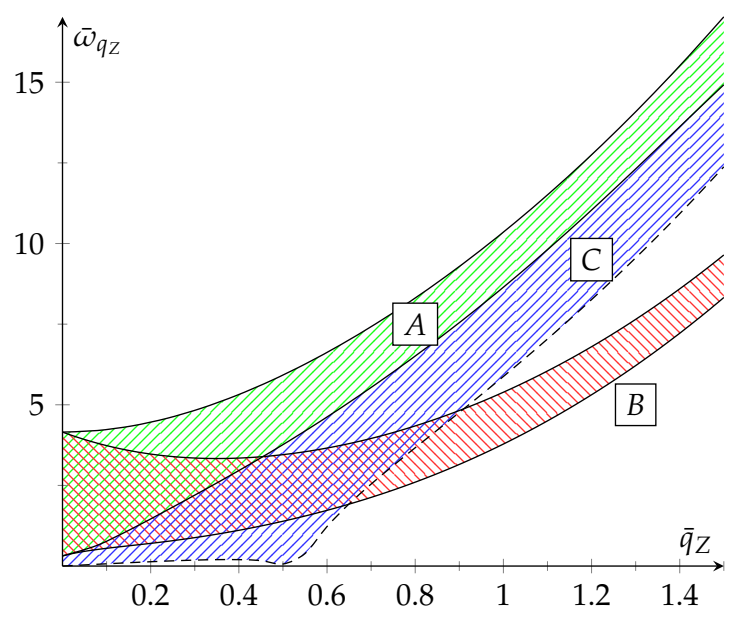

Figura 7. Estructura de bandas para los modos de propagación de las ondas de espín transversales en interacción con la corriente de portadores electrónicos. En todos los casos, $0.01 \leq \omega_{h} / \omega_{D E} \leq 1.5$ y $(A) b_{J_{e}} / \omega_{D E}=0.0, c_{J_{e}} / \omega_{D E}=0.0 ;(B)$ $b_{J_{e}} / \omega_{D E}=5, c_{J_{e}} / \omega_{D E}=1.5 ;(C) b_{J_{e}} / \omega_{D E}=-5, c_{J_{e}} / \omega_{D E}=$ -1.5 .

unitario $\Omega^{-1}$ :

$$
\langle n\rangle=\frac{\pi^{3 / 2}}{(\hbar \beta \mathcal{D}(J))^{3 / 2}} \exp \left[-\hbar \beta\left(\omega_{H}-\frac{b_{J_{e}}^{2}}{4 \mathcal{D}(J)}\right)\right]
$$

el cual conduce a la relación clásica de Bloch $\langle n\rangle \sim T^{3 / 2}$ en el caso $J_{e}=0 \mathrm{y} \omega_{H}=0$. El tiempo medio de emisión de ondas de espín en función de la temperatura es una relación lineal del tipo $\left\langle\tau^{-1}\right\rangle=\tau_{0}^{-1}+3 \alpha \hbar^{-1} k T \mathrm{y}$ $\tau_{0}^{-1}=2 \alpha \omega_{H}+\left(2 \tau_{e x} / \alpha \tau_{S F}-1\right) \alpha b_{J_{e}}^{2} / 2 \mathcal{D}(J)$. El diagra- 


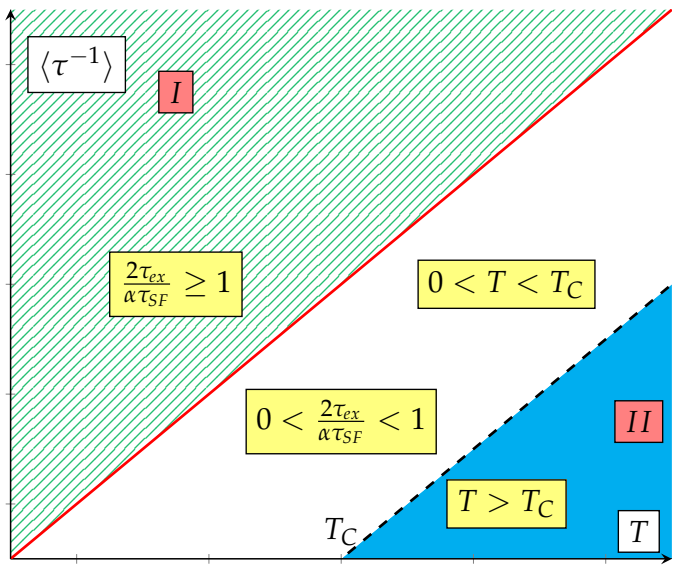

Figura 8. Diagrama de fases asociado al inverso del tiempo de vida promedio de las excitaciones de ondas de espín en función de la temperatura absoluta $T$ y el parámetro característico $2 \tau_{e x} / \alpha \tau_{S F}$.

ma de fases $\left[\left\langle\tau^{-1}\right\rangle, T\right]$ se describe en la Figura (8). El parámetro $2 \tau_{e x} / \alpha \tau_{S F}$ define dos tipos de excitaciones: (I) ondas de espín inducidas (ISW) con tiempo de vida restringido a un valor de temperatura finito bajo la condición $2 \tau_{e x} / \alpha \tau_{S F} \geq 1, \mathrm{y}(I I)$ ondas de espín térmicamente generadas a temperaturas mayores que cierto valor crítico $T_{C}$, bajo la condición $0<2 \tau_{e x} / \alpha \tau_{S F}<1$. En este caso, el tiempo de emisión es mayor a temperatura fija comparado con los tiempos característicos asociados a la fase (I). Ondas coherentes de espín (CSW) con $\left\langle\tau^{-1}\right\rangle=0$ surgen en este régimen bajo la condición: $k T_{C}=\left(1-2 \tau_{e x} / \alpha \tau_{S F}\right) b_{J_{e}}^{2} / 6 \hbar^{-1} \mathcal{D}(J)$, con $\omega_{H}=0$. Este diagrama de fases esquemático resalta el rôle del factor de Gilbert en la configuración de los modos de propagación, la estabilidad, el tiempo medio de vida de ondas emitidas y su correlación con la temperatura: el valor crítico de $\alpha$ es del orden de $2 \tau_{e x} / \tau_{S F}$, el cual define la escala de prevalencia en los efectos de relajación de las fluctuaciones de la magnetización transversal $\left(\tau_{S F}\right)$, sobre el mecanismo de interacción entre los electrones itinerantes y electrones localizados $\left(\tau_{e x}\right)$. El diagrama de fases indica que no es posible la emisión de ondas transversales de espín en el rango $0<T<T_{C}$ con $2 \tau_{e x} / \alpha \tau_{S F}<1$.

\section{Condición de criticalidad en 1D}

La ecuación (8) tiene una solución en términos del propagador compuesto $K_{\infty}^{ \pm}\left(z, z^{\prime} ; t\right)$ (para un medio infinitamente extendido):

$$
\begin{array}{r}
m^{ \pm}(z, t)=\int_{-\infty}^{\infty} K_{\infty}^{ \pm}\left(z, z^{\prime} ; t\right) m^{ \pm}\left(z^{\prime}, 0\right) d z^{\prime}+ \\
\int_{0}^{t} \int_{-\infty}^{\infty} K_{\infty}^{ \pm}\left(z, z^{\prime} ; t-\tau\right) F^{ \pm}\left(z^{\prime}, \tau\right) d z^{\prime} d \tau,
\end{array}
$$

$\operatorname{con} K_{\infty}^{ \pm}\left(z, z^{\prime} ; t\right)$ igual a:

$$
K_{\infty}^{ \pm}\left(z, z^{\prime} ; t\right)=\frac{1}{2 \sqrt{\pi a_{ \pm}^{2} t}} \exp \left[-\frac{G^{ \pm}\left(z, z^{\prime} ; t\right)}{4 a_{ \pm}^{2} t}\right],
$$

y $G^{ \pm}\left(z, z^{\prime} ; t\right)=\left(z-z^{\prime}+b_{ \pm} t\right)^{2}-4 a_{ \pm}^{2} t^{2} \bar{\omega}_{H}^{ \pm}$. La función $K_{\infty}^{ \pm}$reproduce la solución de la ecuación del calor en una dimensión, en ausencia de corriente externa aplicada $\left(b_{ \pm}=0\right)$ y $\omega_{H}=0^{2}$. En la representación del momentum y la frecuencia (en notación reducida), $K_{\infty}(i v, p)$ tiene la forma:

$$
K_{\infty}(i v, p)=\frac{1}{2 \pi} \frac{1}{2 a^{2} \sigma(i v)} \frac{\sigma^{\prime}(i v)}{\left(\sigma^{\prime 2}(i v)+p^{2}\right)},
$$

con las funciones $\sigma^{\prime}(i v)=\sigma(i v)+b / 2 a^{2}, a \sigma(i v)=$ $\xi\left(i v-\bar{\omega}_{H}-i b^{2} / 4 a^{2}\right)^{1 / 2}, \xi=i^{1 / 2}$. En el caso $b=\bar{\omega}_{H}=$ 0 , la expresión (19) concuerda con la función de correlación de las fluctuaciones transversales de la magnetización $\left\langle m^{ \pm}(p, i v) m^{ \pm}(-p,-i v)\right\rangle$ :

$$
-i K_{\infty}(i v, p) \sim \frac{1}{\mathcal{D}(J) p^{2}-i v},
$$

demostrando el carácter estrictamente difusivo de estas perturbaciones. La presencia de una corriente externa modifica la naturaleza de la propagación de las fluctuaciones magnéticas hacia un régimen intermedio difusivoondulatorio, con una velocidad de arrastre proporcional a la magnitud de $b_{ \pm}$, y a su vez asociada a las distorsiones de la pared de dominio (Yamaguchi A., 2004). Si se aplica un pulso magnético instantáneo $H(t)=H_{0} \delta(t)$ que da lugar a un campo espectral de ruido blanco $H(i v)=H_{0}$, la corrección en la susceptibilidad estática $(i v \rightarrow 0)$ en una dimensión converge para $\sigma^{\prime}(0) \sigma(0) \neq 0$, y está definida para todos los posibles valores del momentum de las ondas de espín por:

$$
\Delta \chi(0) \propto \int_{-\infty}^{\infty} \frac{d p}{2 \pi} K_{\infty}(0, p)=\frac{1}{8 \pi a^{2} \sigma(0) \sigma^{\prime}(0)} .
$$

En el caso $\sigma(0)=0, \omega_{H}=b_{J_{e}} c_{J_{e}} / 2 \mathcal{D}(J)$ y $\sigma^{\prime}(0)=0$, $\omega_{H}=b_{I_{e}} c_{I_{e}} / \mathcal{D}(J)$ cuyas soluciones tienen la estructura $J_{e C} \sim H_{0 Z}^{1 / 2}$. Para frecuencias diferentes de cero, la ecuación (21) se debe modificar como:

$$
\int_{-\infty}^{\infty} \frac{d p}{2 \pi} K_{\infty}(i v, p)=\frac{1}{8 \pi a^{2} \sigma(i v)} .
$$

\footnotetext{
${ }^{2}$ En este resultado deben considerarse las identidades, con $K_{\infty} \equiv$ $K_{\infty}\left(z, z^{\prime} ; t-\tau\right)$ :$$
\partial_{\tau} K_{\infty}+a^{2} \partial_{z^{\prime} z^{\prime}}^{2} K_{\infty}-b \partial_{z^{\prime}} K_{\infty}+\bar{\omega}_{H} K_{\infty}=0
$$$$
\text { y } K_{\infty}\left(z, z^{\prime} ; t-\tau \rightarrow 0\right)=\delta\left(z-z^{\prime}\right) \text {. }
$$ 
La suma sobre las frecuencias complejas de Matsubara iv (Bosónicas) permite obtener la dependencia térmica de la corrección de la susceptibilidad $\Delta \chi$ :

$$
\Delta \chi(T) \sim \frac{1}{8 \pi a^{2}} \sum_{i v} \frac{1}{\sigma(i v)}=\frac{1}{8 \pi a^{2}} \frac{1}{2 \pi i} \oint_{\mathcal{C}} \frac{n_{B E}(z)}{\sigma(z)} d z,
$$

en donde $n_{B E}(z)=(\exp [\beta z]-1)^{-1}$ es el factor estadístico de Bose-Einstein. El resultado de la integración conduce a (Lavrik A. F., 2011):

$$
\Delta \chi(T) \sim \frac{\xi}{16 \pi^{2} a} \Gamma\left(\frac{1}{2}\right) \operatorname{Li}_{\frac{1}{2}}(\exp (-\beta w))
$$

$w=\bar{\omega}_{H}+i b^{2} / 4 a^{2}$ y $\operatorname{Li}_{n}(z)$ como la función Polilogarítmica de orden $n$ con argumento $z$ (Función $\mathrm{Li}_{n}$ ). La componente real de $\operatorname{Li}_{\frac{1}{2}}(z)$ es indefinida en $z=1$ $(w=0)$ y en consecuencia, una relación crítica del tipo $\left|J_{e C}\right| \sim\left|H_{0 Z}\right|^{1 / 2}$ surge bajo este criterio. Esta relación coincide en general con aquella obtenida en el régimen de frecuencia cero.

\section{Resumen y conclusiones}

En este artículo se presenta un análisis del efecto de la interacción de la corriente de portadores electrónicos sobre las ondas de espín en un medio metálico en fase ferromagnética. El término de acople entre la magnetización local y la corriente externa es del tipo torque inducido T [Ec. (3)]. La solución a la ecuación LLGB resultante es estudiada utilizando una variante para el propagador de difusión en sistemas infinitamente extendidos en 1D y $3 \mathrm{D}$, mientras que el efecto de las fronteras de contacto es analíticamente considerado en el caso más general posible en 1D y bajo la prescripción de una solución del tipo Dirichlet (Apéndice I). Diferentes resultados físicos son obtenidos y discutidos, en particular: (i) la variación de estructura de bandas asociadas a las energías de los modos colectivos del tipo Damon-Esbach bajo los efectos de la corriente de portadores, (ii) el tiempo de vida de las ondas de espín estimuladas vía fuente externa (campo magnético o corriente de portadores de carga), su dependencia con los tiempos característicos $\tau_{S F}, \tau_{e x}$ y el factor de Gilbert $\alpha$ en la aproximación de la distribución de Boltzmann, y (iii) la relación de inestabilidad para las ondas de espín en términos de la densidad de corriente de portadores de carga y el campo magnético exterior desde la respuesta de susceptibilidad estática en sistemas 1D. Estos resultados permiten plantear problemas más específicos y de interés tecnológico relacionados por ejemplo con la emisión coherente de magnones en dispositivos híbridos de contacto del tipo grafito laminar/ferromagneto (Tang Z. et-al, 2013), considerando las funcionales de la acumulación de la densidad de portadores de espín en las superficies de frontera (tomadas como cero en este desarrollo), y diferentes estados de polarización de la corriente y el campo exterior en el rango de la radiación de microondas. Es necesario resaltar que el tratamiento presentado en este artículo es de carácter semiclásico. Una derivación rigurosa y muy reciente de la naturaleza gauge del efecto Hall de espín (SHE) puede encontrarse en (Tan S. G. et-al, 2015).

\section{Apéndice I: solución tipo Dirichlet en 1D}

Introduciendo las definiciones $\mathbf{M}=\left(m^{X}, m^{Y}, M_{0}\right)$, $F^{ \pm}=\mp i \gamma M_{0} H_{0 Z} /(1 \mp i \alpha)$, la ecuación (2) toma la forma:

$$
\frac{\partial m^{ \pm}}{\partial t}=a_{ \pm}^{2} \frac{\partial^{2} m^{ \pm}}{\partial z^{2}}+b_{ \pm} \frac{\partial m^{ \pm}}{\partial z}+\bar{\omega}_{H}^{ \pm} m^{ \pm}+F^{ \pm} ; \quad 0<z<\ell,
$$

con $m^{ \pm}=m^{X} \pm i m^{Y}$. La solución para las componentes $m^{ \pm}$se obtienen desde la transformación:

$$
\begin{aligned}
m^{ \pm}(z, t)=u^{ \pm}(z, t)+\left(1-\frac{z}{\ell}\right) & m^{ \pm}(0, t) \\
+ & \frac{z}{\ell} m^{ \pm}(\ell, t),
\end{aligned}
$$

válida para $t>0,0<z<\ell$, en donde $m^{ \pm}(0, t)$ y $m^{ \pm}(\ell, t)$ corresponden a las condiciones de frontera en $z=0$ y $z=\ell$ respectivamente, y $u^{ \pm}(0, t)=u^{ \pm}(\ell, t)=0$. La ecuación (26) debe reescribirse como:

$$
\frac{\partial u^{ \pm}}{\partial t}=a_{ \pm}^{2} \frac{\partial^{2} u^{ \pm}}{\partial z^{2}}+b_{ \pm} \frac{\partial u^{ \pm}}{\partial z}+\bar{\omega}_{H}^{ \pm} u+\bar{F}^{ \pm} ; 0<z<\ell
$$

$t>0$. El término fuente se escribe ahora como:

$$
\begin{aligned}
\bar{F}^{ \pm}(z, t) & =F^{ \pm}+\frac{b_{ \pm}}{\ell}\left(m^{ \pm}(\ell, t)-m^{ \pm}(0, t)\right) \\
-\left(1-\frac{z}{\ell}\right)\left(\frac{\partial m^{ \pm}(0, t)}{\partial t}\right)-\frac{z}{\ell}\left(\frac{\partial m^{ \pm}(\ell, t)}{\partial t}\right)+ & \\
\bar{\omega}_{H}^{ \pm} & {\left[\left(1-\frac{z}{\ell}\right) m^{ \pm}(0, t)+\frac{z}{\ell} m^{ \pm}(\ell, t)\right] . }
\end{aligned}
$$

La solución completa para $u^{ \pm}(z, t)$ puede escribirse en forma compacta como (Guenther R. B. and Lee J. W., 1988) (en notación reducida $a \equiv a_{ \pm}, b \equiv b_{ \pm}, F \equiv F^{ \pm}$, $\left.\bar{\omega}_{H}^{ \pm}=\bar{\omega}_{H}\right)$ :

$$
u(z, t)=\frac{2}{\ell} \int_{0}^{\ell} \sum_{n=1}^{\infty} U_{n}\left(z^{\prime}, t\right) \sin \left(\frac{n \pi z}{\ell}\right) \sin \left(\frac{n \pi z^{\prime}}{\ell}\right) d z^{\prime},
$$

con los coeficientes de $U_{n}\left(z^{\prime}, t\right)$ dados por:

$$
\begin{aligned}
& U_{n}\left(z^{\prime}, t\right)=\exp \left[-Q_{n}\left(z-z^{\prime}, t\right)\right] u\left(z^{\prime}, 0\right)+ \\
& \quad \int_{0}^{t} \exp \left[-R_{n}(t-\tau)\right] \bar{F}\left(z^{\prime}, \tau\right) d \tau, \quad 0<\tau<t .
\end{aligned}
$$


Las funciones $Q_{n}(z, t)$ y $R_{n}(t)$ se definen como:

$$
\begin{array}{r}
Q_{n}(z, t)=\left(\frac{a^{2} \pi^{2} n^{2}}{\ell^{2}}-\bar{\omega}_{H}\right) t+\frac{b}{2 a^{2}}\left(z+\frac{b}{2} t\right), \\
R_{n}(t)=Q_{n}(z, t)-\frac{b z}{2 a^{2}} .
\end{array}
$$

El caso particular $b_{ \pm}=\bar{F}^{ \pm}=0$, reduce (29) a la solución típica de la ecuación del calor en una dimensión y condiciones de Dirichlet:

$$
\begin{aligned}
& u^{0}(z, t)= \\
& \frac{2}{\ell} \int_{0}^{\ell} \sum_{n=1}^{\infty} U_{n}^{0}\left(z^{\prime}, t\right) \sin \left(\frac{n \pi z}{\ell}\right) \sin \left(\frac{n \pi z^{\prime}}{\ell}\right) d z^{\prime},
\end{aligned}
$$

$\operatorname{con} U_{n}^{0}\left(z^{\prime}, t\right)=u\left(z^{\prime}, 0\right) \exp \left[-a^{2} \pi^{2} n^{2} t / \ell^{2}\right]$. Con un procedimiento similar, la solución para la densidad electrónica $\mu(z, t)$ puede expresarse como:

$$
\begin{gathered}
\mu(z, t)=v(z, t)+\left(1-\frac{z}{\ell}\right) \mu_{0}(t)+\frac{z}{\ell} \mu_{\ell}(t) ; \\
0<z<\ell, v(0, t)=v(\ell, t)=0 \mathrm{y} \\
v(z, t)= \\
\frac{2}{\ell} \int_{0}^{\ell} \sum_{n=1}^{\infty} \bar{h}_{n}\left(z^{\prime}, t\right) \sin \left(\frac{n \pi z}{\ell}\right) \sin \left(\frac{n \pi z^{\prime}}{\ell}\right) d z^{\prime},
\end{gathered}
$$

y el kernel $\bar{h}_{n}$ definido explícitamente por:

$$
\begin{aligned}
& \bar{h}_{n}(z, t)= \\
& \quad \frac{D_{0}^{-1} H_{J_{e}}(z, t)-\kappa_{i}^{2}\left[(1-z / \ell) \mu_{0}(t)+z \mu_{\ell}(t) / \ell\right]}{\kappa_{i}^{2}-n^{2} \pi^{2} / \ell^{2}} .
\end{aligned}
$$

Las funciones $\mu_{0}(t), \mu_{\ell}(t)$ corresponden a las distribuciones de las densidades de espín electrónico en las superficies $z=0$ y $z=\ell$ respectivamente. La función $H_{J_{e}}(z, t)$ en (35) es, en términos de $\vec{\mu}$ :

$$
\mathbf{H}_{J_{e}}(z, t)=D_{0} \nabla^{2} \vec{\mu}-\frac{1}{\tau_{e x}} \vec{\mu} \times \hat{\mathbf{z}}-\frac{\vec{\mu}}{\tau_{s f}} .
$$

Las componentes de la proyección del campo $H_{J_{e}}(z, t)$ en el plano $X Y$ en la representación de onda de espín rotada (SWRR) son equivalentes a las definidas en la ecuación (5).

\section{Apéndice II}

En notación simplificada, la solución a la ecuación homogénea $\hat{L}^{ \pm} m^{ \pm}=0$ [Cfr. Ec. (8)] toma la forma: $m^{ \pm}(q, t) \sim \exp \left[-M_{q} t\right], \operatorname{con} M_{q}=a_{ \pm}^{2} q^{2}-i b_{ \pm} q \cos \theta-$ $\bar{\omega}_{H}^{ \pm}$. Integrando en el dominio del momentum, se obtiene el resultado (9), con $R=\left|\mathbf{r}-\mathbf{r}^{\prime}\right|$.

$$
\begin{array}{r}
\int_{0}^{\pi} \int_{0}^{\infty} \exp \left[-M_{q} t+i q R \cos \theta\right] q^{2} \sin \theta d \theta d q \\
\sim K_{\infty}^{3 D \pm}\left(\mathbf{r}, \mathbf{r}^{\prime} ; t\right) .
\end{array}
$$

\section{Conflicto de intereses}

$\mathrm{El}$ autor no reconoce conflicto de interés alguno en el proceso de escritura y publicación de este artículo.

\section{Bibliografía}

Anderson P. W. (1961). Localized Magnetic States in Metals, Phys. Rev. 124: 41. doi: 10.1103/PhysRev.124.41

Akhiezer I. A., Bar'yakhtar V. G. and Peletminskii S. V. (1961). On the theory of low-temperature, high-frequency magnetic susceptibility of a ferrodielectric, Soviet Physics JETP 13: 249.

Bazaliy Ya, B. Jones B. A. and Zhang S. -C. (1998). Modification of the Landau-Lifshitz equation in the presence of a spinpolarized current in colossal- and giant-magnetoresistive materials, Phys. Rev. B 57, R3213. doi: 10.1103/PhysRevB. 57.R3213

Berger L. (1996). Emission of spin waves by a magnetic multilayer traversed by a current, Phys. Rev. B 54, 9353. doi: 10.1103/ PhysRevB.54.9353

Chupis I. E. (1964). Theory of Relaxation processes in a uniaxial antiferrodielectric, Soviet Physics JETP 19: 212. doi: http:// www.jetp.ac.ru/cgi-bin/dn/e_019_01_0212.pdf.

Cottam M. and Tilley M. R. (1989). Introduction to Surface and Superlattice Excitations, Cambridge University Press, (1989).

Demidov V. E., Urazhdin S. and Demokritov S. O. (2010) Direct observation and mapping of spin waves emitted by spin-torque nanooscillators, Nature 9: 984. doi: 0.1038/ nmat2882

Eshbach J. R. and Damon R. W. (1960). Surface Magnetostatic Modes and Surface Spin Waves, Phys. Rev. 118: 1208. doi: 10.1103/PhysRev.118.1208

Fetter A. andWalecka J. D. (2002). Quantum Theory of Many Particle Systems, Dover Publications.

Función Lin (z), http://functions.wolfram.com/10.08.02.0001.01

$$
\operatorname{Li}_{n}(z)=\sum_{k=1}^{\infty} \frac{z^{k}}{k^{n}} ; \quad \Gamma\left(\frac{1}{2}\right) \operatorname{Li}_{\frac{1}{2}}\left(e^{\mu}\right)=\int_{0}^{\infty} \frac{k^{-1 / 2} d k}{e^{(k-\mu)}-1} .
$$

Gilbert T. L. (2004). Classics in Magnetics: A Phenomenological Theory of Damping in Ferromagnetic Materials, IEEE Transactions on Magnetics, Vol. 40 (6): 3443. doi: 10.1109/ TMAG.2004.836740

Gómez J., Pérez F., Jusserand B., Karczewzski K. and Wojtowicz T. (2011). Propagation length of spin waves in a conducting system, Journal of Physics: Conference Series 334 012055. doi: 10.1088/1742-6596/334/1/012055

Guenther R. B. and Lee J.W. (1988). Partial Differential Equations of Mathematical Physics and Integral Equations, Dover Publications.

Hertz J. and Edwards D. M. (1973). Electron-magnon interactions in itinerant ferromagnetism: Formal theory, J. Phys. F: Metal Phys., 3 2174. doi: 10.1088/0305-4608/3/12/018 
Kitamura Y., Shikoh E., Ando Y., Shinjo T., Shiraishi M. (2013). Vertical spin transport in $\mathrm{Al}$ with $\mathrm{Pd} / \mathrm{Al} / \mathrm{Ni} 80 \mathrm{Fe} 20$ trilayer films at room temperature by spin pumping, M. Sci. Rep. 3, 1739; doi: 10.1038/srep01739

Kondo J. (1964). Resistance Minimum in Dilute Magnetic Alloys, Prog. Theor. Phys. 2 (1): 37-49. doi: 10.1143/PTP.32.37

Lavrik A. F. Complex integration, method of. A.F. Lavrik (originator), Encyclopedia of Mathematics. URL: http:// www.encyclopediaofmath.org.

Li Z., He J. and Zhang S. (2006). Effects of spin current on ferromagnets, Journal of Applied Physics 99 08Q702. doi: $10.1063 / 1.2166590$

Maekawa S., Adachi H., Uchida K., Ieda J. and Saitoh E. (2013). Spin Current: Experimental and Theoretical Aspects, Journal of the Physical Society of Japan 82 102002. doi: 10.7566/JPSJ.82.102002

Madami M., Bonetti S., Consolo G., Tacchi S., Carlotti G, Gubbiotti G., Mancoff F. B., Yar M. A. and Akerman J. (2011). Direct observation of a propagating spin wave induced by spin-transfer torque, Nature Nanotechnology 6: 635 . doi: 10.1038/nnano.2011.140

Phillips T. G. and Rosenberg H. M. (1966). Spin Waves in Ferromagnets, Rep. Prog. Phys. 29 285. doi: 10.1088/0034$4885 / 29 / 1 / 307$

Saitoh, E., Ueda, M., Miyajima, H. and Tatara, G. (2006). Conversion of spin current into charge current at room temperature: Inverse spin-Hall effect. Appl. Phys. Lett. 88, 182509. doi: 10.1063/1.2199473
Sears M. R. and Saslow W. M. (2012). Spin accumulation at ferromagnet/nonmagnetic material interfaces, Phys. Rev. B 85, 014404. doi: 10.1103/PhysRevB.85.014404

Tan S. G. et al., (2015). Gauge Physics of Spin Hall Effect, Sci. Rep. 5, 18409. doi: 10.1038/srep18409

Tang Z, Shikoh E., Ago H., Kawahara K., Ando Y., Shinjo T., and Shiraishi M. (2013). Phys. Rev. B 87, 140401 (R). doi: 10.1103/PhysRevB.87.140401

Tsoi M., Jansen A. G. M., Bass J., Chiang W. -C, Tsoi V. and Wyder P. (2000).Generation and detection of phasecoherent current-driven magnons in magnetic multilayers, Nature 406, 46. doi: 10.1038/35017512

Yamaguchi A. (2004). Real-Space Observation of Current-Driven Domain Wall Motion in Submicron Magnetic Wires Phys. Rev. Lett. 92, 077205. doi: 10.1103/PhysRevLett.92.077205

Zhang S. and Li Z. (2010). Roles of Nonequilibrium Conduction Electrons on the Magnetization Dynamics of Ferromagnets, Physical Review Letters 93, 127204. doi: 10.1103/ PhysRevLett.93.127204

Zhang S. and Zhang S. S. -L. (2009). Generalization of the LandauLifshitz-Gilbert Equation for Conducting Ferromagnets, Physical Review Letters 102, 086601. doi: 10.1103/ PhysRevLett.102.086601

Žuti'c I., Fabian J., Das Sarma S. (2004). Spintronics: Fundamentals and Applications, Review of Modern Physics 76: 323-414, arXiv:cond-mat/0405528v1. 